\title{
The effect of implementing peripherally inserted central catheter educational program on nurses' knowledge, practice, and neonatal outcomes
}

\author{
Omnia Saltah ${ }^{1}$ and Fawzia Abusaad ${ }^{2}$ \\ ${ }^{1}$ Pediatric Nursing, Faculty of Nursing, Mansoura University, Egypt \\ 2Pediatric Nursing Department, Faculty of Nursing, Mansoura University, Egypt
}

\section{III}

Background: The peripherally inserted central catheters (PICCS) have become among the most common technologies employed in the intravenous therapy of neonates as they allow safe long-term intravascular access, comfort, and ease of transition to home therapy that contributed to the increased survival of those newborns. Aim: This study aimed to evaluate the effect of implementing peripherally inserted central catheter educational program on nurses' knowledge, practice, and neonatal outcomes. Materials and Methods: pretest-posttest quasi-experimental design was conducted on 45 neonatal nurses working at neonatal intensive care units affiliated to Mansoura University Children Hospital (MUCH) with a purposive sample of (20) neonates admitted to NICU throughout six months and need PICC line insertion was enrolled in the study. Tools of data collection: Data were collected by using three tools: Peripherally inserted central catheter insertion questionnaire sheet, PICC line insertion and care observational checklist and neonatal assessment sheet. Results: Majority of studied nurses had poor knowledge and more than half had competent skills about PICC before program implementation while after program implementation there was more than half had average knowledge and two third had competent practices as well as there is decrease in the prevalence of neonatal complication. Conclusion: There was appositive effect of the educational program on nurse's knowledge and skills as well as neonatal. Recommendation: Continuing of nursing education and practices about PICC lines.

Keywords: Educational program; knowledge; Neonates; Neonatal nurse; PICC line

Editor-in-Chief: Prof. M.L. Salem, PhD - Article DOI: 10.21608/JCBR.2021.59939.1137 